\title{
Numerical solution of the spin angular momenta of millisecond pulsar PSR J0751+1807
}

\author{
B.P. Gong and K.S.Cheng \\ Department of Physics, University of Hong Kong, Pokfulam Road, Hong \\ Kong, China
}

\begin{abstract}
The motion of the binary pulsar PSR J0751+1807 is assumed to satisfy the gravitational two-body equation(Barker and O'Connell 1975). Then by studying the motion of the binary system from initial state which determined by 3 precession phases of angular momenta (the orbit, the pulsar and the companion star) to the current state which determined by the observations on the orbital inclination. The spin angular momenta of the pulsar and the companion star are obtained.
\end{abstract}

\section{Introduction}

The parameters on the orbital period $\left(P_{b}=0.263\right.$ days $)$, the mass of the companion star $\left(m_{1}=0.15 M_{\odot}\right)$, the mass of the pulsar $\left(m_{2}=1.4 M_{\odot}\right)$, and the orbital inclination $\left(i=60^{\circ}\right)$ are based on the following observations: Lundgren et al. $(1995,1996)$, Ergma et al. (1997). The binary pulsar PSR $0751+1807$ is treated as a conservative system(the time scale of motion is much smaller than the age of the binary), and the spin axis of the pulsar is treated as very close to orbital angular momentum (standard model of millisecond pulsar formation). Furthermore, by imposing theoretical(the gravitational two-body equation) and observational constraints on the motion of the binary system. The spin angular momenta of the pulsar and the companion star are obtained.

\section{Equations of motion}

According to the two-body equation, the orbital angular momentum $\vec{L}$ precess around the total angular momentum $\vec{J}$, the spin angular momenta of the companion star $\vec{S}_{1}$ and the pulsar $\vec{S}_{2}$ precess around $\vec{L}$. The precession phase angles are as follows:

$$
\eta_{i}=\Omega_{i} t+\eta_{i}(0), i=0,1,2
$$

where $\Omega_{0}, \Omega_{1}$ and $\Omega_{2}$ are rates of precession of $\vec{L}$ (orbit), $\vec{S}_{1}$ (companion) and $\overrightarrow{S_{2}}$ (pulsar) respectively. It is likely that the initial phases of precession $\eta_{i}(0)$ can never be known, however, since $\Omega_{0}, \Omega_{1}$ and $\Omega_{2}$ are not equal, after a certain period of time, these 3 precession phases can reach certain values. Any relative value among $\eta_{0}(0), \eta_{1}(0)$ and $\eta_{2}(0)$ are physically possible. Then we choose an arbitrary set of $\eta_{i}$ as the initial phases of motion(at $t=0$ ). Starting from this set of initial state (represented by the initial phases), the binary system can evolve to the current state (represented by $i$ ) at the time $t$. 


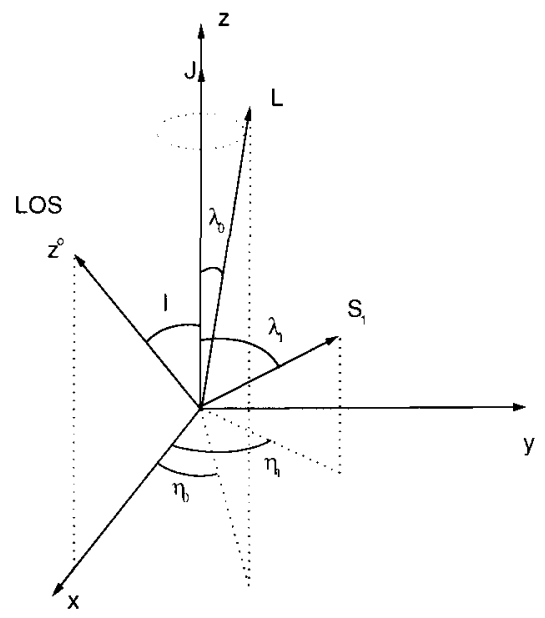

Fig. 1

Figure 1. Coordinate systems to describe geodesic precession. Vector $J, L$ and $Z^{\circ}$ represents the invariable plane, the orbital plane; and the plane corresponding to the line of sight respectively.

The the binary system satisfies the following constraints at both the initial and current state: firstly given by $\vec{J}=\vec{L}+\vec{S}$ and $\dot{J}=0$; Secondly given by the equations of angle transformation among the total angular momentum coordinate system, the orbital angular momentum coordinate and the observational coordinate system.

\section{Result}

By numerical solution of 15 equations obtains the spin angular momentum of the companion star $S_{1}=6.192 \times 10^{46} \mathrm{gcm}^{2} \mathrm{~s}^{-1}$ and the spin angular momentum of the pulsar $S_{2}=1.226 \times 10^{48} \mathrm{gcm}^{2} \mathrm{~s}^{-1}$.

\section{References}

Apostolatos, T.A., Cutler, C., Sussman, G.J., \& Thorne, K.S., 1994, Phys. Rev. D, 49, 6274

Barker, B.M. \& O'Connell, R.F., 1975, Phys. Rev. D., 12, 329

Damour, T. \& Taylor, J.H., 1992, Phys. Rev. D., 45, 1840

Ergma, E., Lundgren, S.C., \& Cordes, J.M., 1997, ApJ, 475, L29

Lundgren, S.C., Cordes, J.M., Foster, R.S., Wolszczan, A., \& Camilo, F., 1996, ApJ, 458, L33

Lundgren, S.C., Zepka, A.F., \& Cordes, J.M., 1995, ApJ, 453, 419 